\title{
INDICADORES EN GEOGRAFÍA SOCIAL
}

POR

\author{
ANA OLIVERA
}

El concepto de indicador social, como presentación cuantitativa de información sobre fenómenos sociales, su localización, desarrollo y correlación, se empleó por primera vez en 1962, por la American Academy of Arts and Sciences. Al final de los sesenta el «movimiento de indicadores sociales» se inició también en Europa y en 1971 la O.C.D.E. comenzó un proyecto de desarrollo de dichos indicadores. Desde entonces los indicadores sociales han estado muy relacionados con todo tipo de políticas y planificación.

Las expectativas que despertaron los indicadores fueron en parte defraudadas, y se desprestigiaron por su ambigüedad y pobreza conceptual. Muchas han sido las críticas contra los indicadores: su simplificación excesiva, su dudosa fiabilidad, la duplicación de información dentro del cálculo de un mismo indicador sintético, la redundancia de indicadores simples en la construcción de varios indicadores sintéticos, el no estar ajustados a la escala de análisis deseable, el precisar un cálculo demasiado complejo, etc. Pero es de esperar que en los próximos años la informatización de grandes bases de datos, que permitan nuevos tipos de referenciación espacial y el avance en el tratamiento estadístico de variables, asicomo el desarrollo conceptual y experimental de los estudios sobre indicadores, tras

Ana Olivera. Universidad Autónoma de Madrid

Estudios Geográfico

Tomo LVIII, n. ${ }^{\circ} 229$, octubre-diciembre 1997 
un nuevo debate sobre su uso pueda producir un enriquecimiento de contenidos y enfoques que beneficie a la Geografía Social.

No es apropiado reducir cualquier análisis a unas cifras, pues ni todo es medible ni basta con medir, ni tampoco demonizar las posibles medidas que nos ayudan a entender, desde el punto de vista espacial, la vida social, y algunos fenómenos (como el estatus de la mujer, la precariedad y temporalidad del trabajo, la vulnerabilidad a los riesgos de salud, etc.) y a definir el carácter social de los espacios, su papel y funcionalidad, en los ámbitos local, regional, nacional y global, así como las interaciones socioespaciales.

Es cierto que los fenómenos sociales y las vidas individuales son demasiado complejas para poder simplificarlas en unos pocos indicadores cuantitativos. Pero aún así es mejor contar con algunos datos que sirvan para una interpretación de esa complejidad, el análisis social o como base para la planificación.

\section{Tipologias de indicadores}

Los indicadores pueden ser clasificados atendiendo a una gran diversidad de características.

a) según el carácter de los datos utilizados en su confección:

- cuantitativos (Q I)

- no cuantitativos (N Q I)

Los cuantitativos se obtienen de datos que reflejan las condiciones sociales, económicas, demográficas o medioambientales de un determinado espacio y permiten evaluar su situación y evolución. Los no cuantitativos son descripciones verbales que hacen referencia, por ejemplo, a carencias: existencia o no de políticas para reducir las desigualdades en salud en una región o país, si poseen o no programas de prevención, o de integración de minorías, o métodos de evaluación de calidad de servicios de salud o asistenciales (Nossikov, 1990).

b) según el tipo de medida:

- objetivos

- subjetivos

Los objetivos son los que se obtienen por registro, por ejemplo número y proporción de escolarizados, pensionistas, altas hospitalarias, etc. Son indicadores subjetivos los basados en información obte-

$$
-690-
$$


nida del individuo sobre percepción del estado de bienestar, satisfacción general, salud o seguridad, etc.

c) según su forma de cálculo:

- simples

- compuestos o sintéticos

Los indicadores simples más habituales son: cantidades absolutas o stocks, distribuciones porcentuales, tasas de frecuencia, tasas de cambio y números índice, medidas de tendencia central, número ordinal según valor de una variable). Los sintéticos resumen en una sola cifra el conjunto de varios simples.

d) según variables consideradas:

Existen indicadores de muy diversas temáticas, que pueden ser usados aisladamente o combinados en índices complejos.

- económicos: segmentación salarial, existencia de dobles tablas salariales; trabajo a tiempo completo o parcial, contratación indefinida o temporal, duración media de la jornada laboral; gasto social en educación, salud o pensiones; composición porcentual del consumo doméstico.

- sociales: pobreza (Cuadro I), cobertura por prestaciones sociales; seguridad; derechos humanos; derecho al trabajo y protección frente al desempleo; stock educativo; escolarización en los diversos niveles; proporción de escolarizados en la enseñanza pública y privada y dentro de ésta en la religiosa o en la laica; uso del tiempo; delincuencia.

- demográficos: crecimiento natural, fecundidad total, fecundidad adolescente, acceso a la planificación familiar, proporción de nacidos con bajo peso; inmigración; envejecimiento; patrones de mortalidad, mortalidad infantil, mortalidad del grupo 1 a 5 años, incidencia del suicidio.

- culturales: proporción de autores, editores, librerías, bibliotecas y su frecuentación, utilización o niveles de lectura.

- de salud: patrones de morbilidad, prevalencia e incidencia de enfermedades sociales, crónicas (Fig. 1), infecciosas y SIDA; mortalidad evitable, años potenciales de vida perdidos según causa, mortalidad materna; discapacidades, esperanza de vida libre de incapacidad; esperanza de vida en buena salud; grado de inmunización de la población infantil; indicadores de dotación de equipamientos y servicios, y técnicas de diagnóstico, accesibilidad a centro hospitalario (Cuadro II).

- medioambientales: población que tiene acceso a agua pura, saneamiento; deforestación, uso de recursos naturales, porcentaje de reciclado de basuras. 
CuAdro I

PORCENTAJE DE HOGARES QUE SE CONSIDERAN CASI POBRES O POBRES. COMUNIDADES AUTÓNOMAS, 1991

\begin{tabular}{|c|c|}
\hline CC.AA. & $\%$ \\
\hline 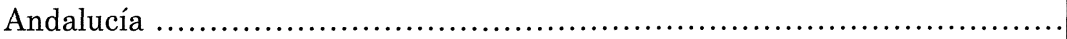 & 16,8 \\
\hline Aragón $\ldots \ldots \ldots \ldots \ldots \ldots \ldots \ldots \ldots \ldots \ldots$ & 2,2 \\
\hline 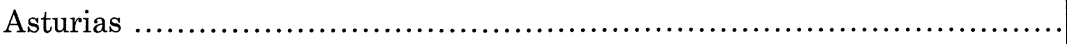 & 6,4 \\
\hline Baleares $\ldots \ldots \ldots \ldots \ldots \ldots \ldots \ldots \ldots$ & 7,8 \\
\hline 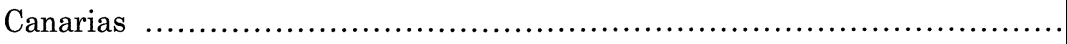 & 19,7 \\
\hline 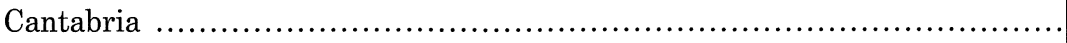 & 3,3 \\
\hline Castilla-León $\ldots . . \ldots \ldots \ldots \ldots \ldots \ldots$ & 5,8 \\
\hline Castilla-La Mancha $. . . . . . . . . . . \ldots . .$. & 12,3 \\
\hline 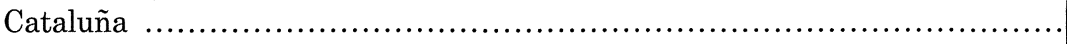 & 5,6 \\
\hline C. Valenciana.................... & 8,9 \\
\hline Extremadura ...................... & 12,8 \\
\hline Galicia $\ldots . \ldots \ldots \ldots \ldots \ldots \ldots \ldots \ldots \ldots$ & 4,5 \\
\hline 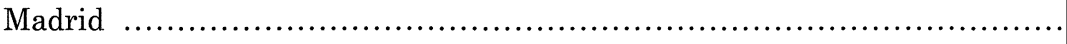 & 5,0 \\
\hline 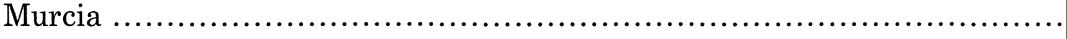 & 7,2 \\
\hline Navarra ........................... & 4,5 \\
\hline 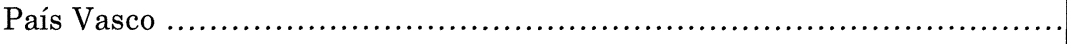 & 5,9 \\
\hline 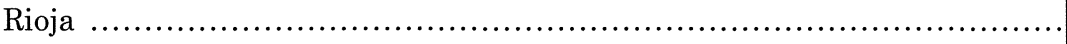 & 4,1 \\
\hline
\end{tabular}

Fuente: I.N.E. Indicadores Sociales de España. 1997.

e) según escalas espaciales ${ }^{1}$ :

- macro (grandes regiones mundiales, países)

- meso (regiones, provincias)

- micro

- local (núcleo)

- infralocal (distrito sanitario, barrio, manzana, espacio interior o medio construido)

f) según escalas sociales y categorias

La información estará clasificada según clases sociales, grupos (género, discapacidad, vejez, raza, etnia) o individuos.

1 Existe confusión entre la terminología cartográfica y la espacial, que es la utilizada generalmente en ciencias sociales, ya que los mapas a macro escala o mapas a gran escala se refieren a micro escala espacial (parcelarios urbanos). 
Andalucía

Aragón

Asturias

Baleares

Canarias

Cantabria

Castilla-León

Castilla-La Macha

Cataluña

Comunidad Valenciana

Extremadura

Galicia

Madrid

Murcia

Navarra

País Vasco

Rioja

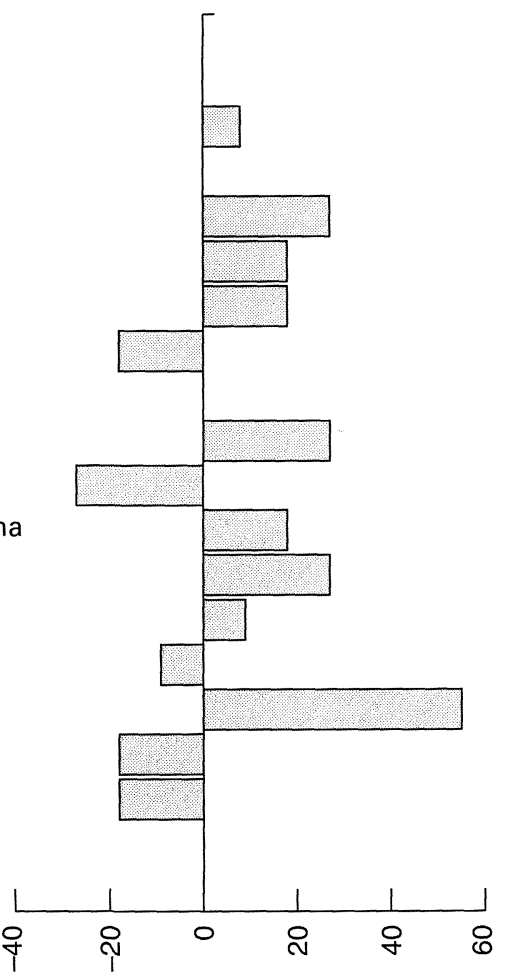

Fuente: Ministerio de Sanidad: Encuesta Nacional de Salud, 1993.

Figura 1.-Enfermedades crónicas diagnosticadas. Hipertensión (\% sobre media nacional).

g) según su relación con el tiempo:

- fijos o sincrónicos

- dinámicos (que resumen crecimientos o marcan tendencias).

h) según escalas temporales

Los datos diacrónicos son registrados en series anuales, mensuales, semanales o diarias, que permiten conocer un proceso en esos determinados saltos temporales. Otras veces son resultado de encuestas esporádicas ${ }^{2}$.

2 Lo ideal en Geografía Social sería poder sumar información de procesos, de acontecimientos aislados, de hechos permanentes y de hechos efímeros, contando con indicadores que facilitaran un «zoom espacio-temporal»: de lo global a lo micro, de lo permanente a lo efímero o eventual, y viceversa. Muchos hechos eventuales son culminación de un proceso. 


\section{Cuadro II}

TASA ESTANDARIZADA DE AÑOS POTENCIALES

DE VIDA PERDIDOS / 1.000 HABITANTES.

MEDIA 1989-90-91

\begin{tabular}{|c|c|}
\hline CC.AA. & Tasa APVP \\
\hline Andalucía $. . . . . . . . . . \ldots \ldots \ldots . . .$. & 49 \\
\hline 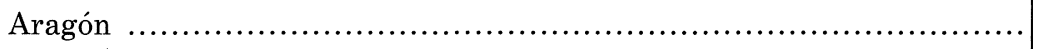 & 44 \\
\hline 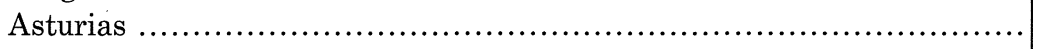 & 52 \\
\hline 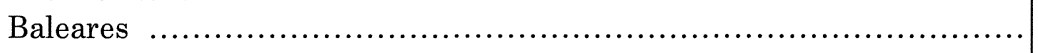 & 58 \\
\hline 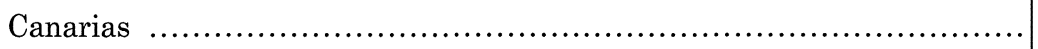 & 52 \\
\hline 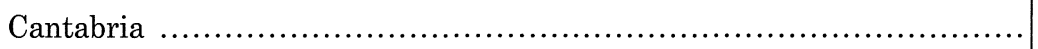 & 49 \\
\hline 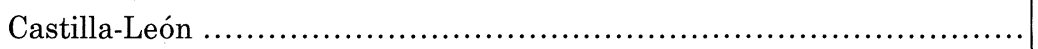 & 44 \\
\hline Castilla-La Mancha .................. & 42 \\
\hline 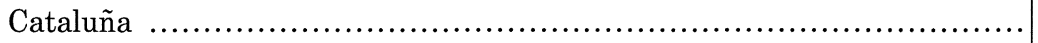 & 50 \\
\hline 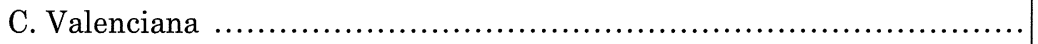 & 51 \\
\hline 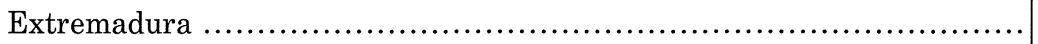 & 46 \\
\hline 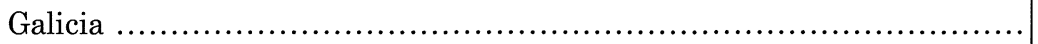 & 52 \\
\hline 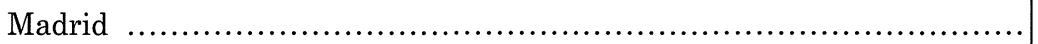 & 50 \\
\hline Murcia .............................. & 50 \\
\hline 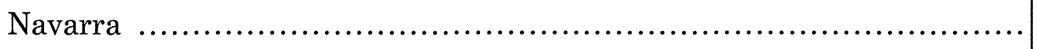 & 41 \\
\hline 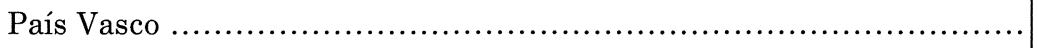 & 49 \\
\hline 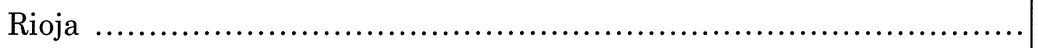 & 47 \\
\hline
\end{tabular}

Fuente: I.N.E. Defunciones según causa de muerte. 1989, 1990, 1991.

Recientes hechos que han influido en la creciente producción de indicadores

A partir de la segunda mitad de la década de los ochenta se ha producido un cambio en la cantidad, calidad y tipo de indicadores. Cada vez son más abundantes y con variables mejor ponderadas, y algunos de ellos permiten mayor desagregación espacial o bien ofrecen datos mundiales sobre aspectos novedosos.

Los cambios temáticos han sido también profundos. Se produjo un desplazamiento desde los indicadores de tipo económico a los económico-sociales, y posteriormente a los sanitarios, de género y medioambientales en relación con población. Se fue pasando del interés por la salud económica de los espacios a la preocupación por el bienestar y salud de su población. 
Varias han sido las circunstancias que han dado lugar a los nuevos intereses temáticos y multiescalares y al nacimiento de múltiples observatorios, dedicados a la medición y seguimiento de indicadores.

1. Se hace cada vez más patente el crecimiento de la polarización social a nivel mundial ${ }^{3}$. Muestra de ello es que entre 1960 y 1993 no sólo no disminuyó la diferencia en el ingreso per cápita entre el mundo industrializado y el mundo en desarrollo sino que se triplicó, pues pasó de 5.700 dólares a 15.400. Muchas situaciones socioespaciales ponen de manifiesto que no siempre existe una relación clara entre crecimiento económico y desarrollo humano, pues en espacios con indicadores económicos muy positivos y fuertes crecimientos no mejoran o incluso pueden empeorar las condiciones de vida de sus habitantes o de una gran parte de ellos (PNUD, 1996). El «Informe sobre desarrollo humano 1996» tuvo la función de derribar dos mitos: el primero es que la prosperidad de los países en desarrollo está favoreciendo la convergencia entre los ricos y los pobres; el segundo es que las primeras etapas de crecimiento económico, inevitablemente, están asociadas con una creciente desigualdad dentro de cada país. Realmente no siempre es así.

Se cuestiona el crecimiento económico como fin en sí mismo y en múltiples foros internacionales se acepta ${ }^{4}$, al menos teóricamente, que el fin del crecimiento económico debería ser enriquecer la vida de la población, conseguir el desarrollo humano, reducir y erradicar la pobreza, lograr el acceso universal a la salud y la educación, nivelar las desigualdades y lograr la sustentabilidad a largo plazo, pues no es deseable un crecimiento sin empleo, sin equidad, sin voz de las comunidades, sin raíces y sin pensar en las generaciones futuras y en el medio ambiente que heredarán. $\mathrm{Ni}$ el crecimiento económico ni los avances en desarrollo humano resultan sostenibles a largo plazo si el uno se produce en ausencia del otro.

3 Por ejemplo, en el período 85-90 la probabilidad de morir antes de los 5 años en las regiones menos desarrolladas era 7 veces mayor que en las más desarrolladas, en comparación con la cifra 6.2 del decenio anterior.

${ }_{4}$ Cumbre Mundial sobre la infancia, 1990; Segunda Conferencia sobre países menos adelantos, 1990; Conferencia sobre Medio Ambiente y Desarrollo Río de Janeiro, 1992; Conferencia Europea contra la Exclusión Social Copenhague, 1993; Conferencia sobre Población y Desarrollo El Cairo, 1994; Cumbre Mundial sobre Desarrollo Social Copenhague, 1995; Cuarta Conferencia Mundial sobre la Mujer, Pekín, 1995; Segunda Conferencia sobre Asentamientos Humanos Habitat II Estambul, 1996. 
Como consecuencia de ello los organismos internacionales, los Estados y algunas organizaciones no gubernamentales se centran en la búsqueda de nuevos indicadores sensibles para el análisis detallado de la situación, que permita la toma de decisiones sobre políticas que respondan a estos planteamientos.

2. Se intenta identificar niveles de bienestar humano, como una condición variable en el espacio. Para ello se desglosan los componentes del bienestar, seleccionando los indicadores socioterritoriales que puedan reflejar la situación y la distribución espacial. Los indicadores tradicionales de bienestar eran económicos, basados en consumo de bienes y servicios y en el proceso productivo (PIB/p.c.), luego se utilizaron los perceptivos, que autovaloraban el grado de felicidad o satisfacción y desde los años 60 se centraron en la calidad de vida, mediante indicadores sociales, incluyendo salud física y mental. Para Smith (1980) la felicidad depende del nivel de satisfacción de tres tipos de necesidades: tener (economía y vivienda), amar (compañía, solidaridad, pertenencia e identidad) y ser (autorealización, trabajo, educación, salud), por lo que los indicadores de bienestar deberían incluir variables sobre calidad de vida material, física, mental e incluso espiritual (paz, participación), que pusieran de manifiesto las policarencias.

Los estudios sobre bienestar precisan indicadores sobre: satisfacción residencial, salud, situación económica, estado social (delincuencia, malos tratos, accesibilidad y seguridad en el empleo, protección social), educación (fracaso escolar, escolarización de 16 a 24 años, jóvenes fuera del sistema educativo y del mercado laboral), dotación de servicios y equipamientos...

3. Nuevos proyectos y programas como los de Ciudades Sostenibles y Ciudades Saludables están padeciendo la carencia y falta de adecuación de indicadores que muestren los diversos niveles al respecto, lo que ha creado la necesidad de nuevos datos locales, que permitan señalar prioridades de inversión y de actuación.

La sostenibilidad implica preservar el capital natural menteniendo la salud pública, la calidad del aire, agua y suelo, con nivel suficiente para preservar la vida y el bienestar humano de forma permanente y con justicia social. La realización y evaluación de un urbanismo preventivo (efecto en salud y en seguridad) reclama un tipo de indicadores basados en microdatos y confeccionados a microescala. 
La preocupación por la urgente necesidad de indicadores válidos para este tipo de estudios fue palpable en la XX Conferencia Internacional de Estadísticas Regionales y Urbanas, celebrada en Madrid en 1996, que dedicó una ponencia a indicadores de desarrollo de ciudades sostenibles. El interés demostrado hace prever un aumento de indicadores y una mejora en los existentes.

Los indicadores de medio ambiente son cada vez más abundantes y suelen publicarse unidos a indicadores sociales o incluso son utilizados ambos conjuntamente para cálculo de algunos indicadores complejos. Pobreza, supervivencia y degradación del medio ambiente están a veces muy entrelazados, pues la degradación del suelo es tanto un resultado como una causa de la marginación social (Watts, 1989), y la pobreza es la principal causa de deterioro del medio ambiente, pues las regiones pobres no tienen otra alternativa que sobreexplotar sus recursos naturales.

4. Los indicadores de salud, que hasta hace pocos años tenían sólo utilidad en análisis sanitarios y demográficos, actualmente son muy empleados como indicadores socioeconómicos indirectos, o bien se combinan con otros tipos de indicadores en la construcción de índices sociales complejos, que intentan medir el bienestar de la población (Olivera, 1993 y 1996). Son un ejemplo de lo que algunos autores han denominado «metadatos».

La inclusión de los temas de salud y salud reproductiva en la Conferencia Internacional sobre Población y Desarrollo de El Cairo en 1994 (Capítulos VII y VIII del Programa de Acción) fue algo totalmente novedoso y planteó por primera vez la necesidad de una salud y derechos reproductivos para la población (Abellán, 1994). Los estudios previos y el desarrollo de los acuerdos adoptados valorizan la conveniencia y utilidad de nuevos indicadores.

El Programa Salud Para Todos en el año 2000, de la Organización Mundial de la Salud, que tiene como finalidad luchar por la equidad en el estado de salud, propuso indicadores globales sobre estado de salud: mortalidad infantil, esperanza de vida al nacer, peso al nacer y relación peso y edad infantil. También ha promovido el desarrollo de indicadores que permitieran conocer el estado de salud de la población con mayor precisión y sus tendencias, en especial en temas relacionados con algunas enfermedades infecciosas, e igualmente la provisión de recursos sanitarios y políticas sanitarias existentes. 
5. Surgen nuevos movimientos sociales, defensores de derechos de grupos específicos, no ya de clase («non class-based»). Entre ellos el movimiento feminista, que abrió nuevos horizontes a la mujer, pero también a las ciencias sociales.

Las estadísticas de Naciones Unidas referidas al período 1975-85 pusieron de manifiesto que las mujeres, que significan la mitad de la población mundial, realizaban los dos tercios de las horas de trabajo en el mundo, y sin embargo sólo se les contabiliza como un tercio del total de la fuerza del trabajo. Sólo ganaban una décima parte de los ingresos monetarios del mundo y eran titulares de una centésima parte de las propiedades, a la vez que aumentaba su responsabilidad en el cuidado de otras personas (García Ramón, 1989). En palabras del PNUD, las mujeres cuentan pero no son contadas.

Comienzan los indicadores de diferenciación socioespacial por género y sus cambios temporales (participación de la mujer en la economía y peso relativo en determinadas profesiones, en los parlamentos, tasas de escolarización femenina y porcentaje de mujeres en trabajos educativos), algunos son datos que intentan medir el papel de las mujeres en el desarrollo y las interrelaciones entre el estatus de la mujer, su peso en la economía y los cambios demográficos (Naciones Unidas, 1989), incidiendo especialmente en las variables de nivelación de sexos. Otros reflejan la inadmisible situación de la mujer en los países en desarrollo, mediante indicadores como la mutilación genital femenina (Fig. 2).

Recientemente otras categorías de población cuentan también con indicadores específicos, como por ejemplo los discapacitados (Cuadro III). La discapacidad, en variación progresiva con la ancianidad, es un factor de exclusión social y espacial de primer orden, ya que en muchos casos excluye de la educación, del trabajo, del acceso al ocio y la cultura, o del uso del espacio público callejero, debido a las barreras urbanísticas, de edificación y de transporte (Olivera y Abellán, 1994). El envejecimiento de la población y sus importantes consecuencias sociales y económicas han sido también factor desencadenante en la formación de indicadores propios para el análisis de su problemática socioespacial (Abellán, 1996).

Los niños y jóvenes son grupos de población que cuentan con diversos indicadores específicos a nivel mundial, y empiezan a aparecer indicadores a nivel regional, como por ejemplo los referidos a los 


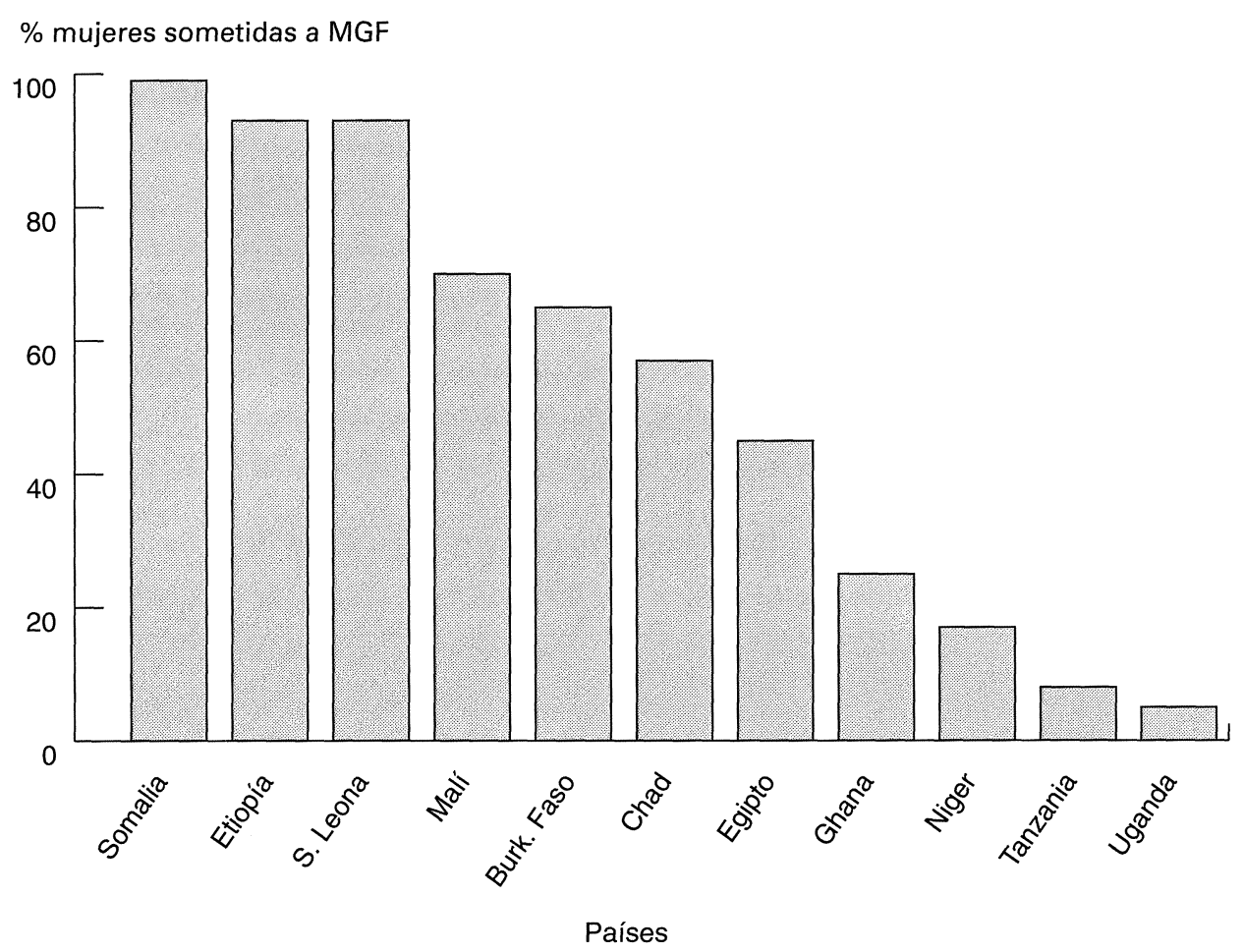

Fuente: Nahid Toubia. Female Genital Mutilation.

Figura 2.-Mutilación genital femenina, 1993.

estados de los Estados Unidos elaborados por la Fundación Annie E. Casey, que contempla tasas de arresto por violencia juvenil, fracaso escolar, porcentaje de jóvenes fuera del sistema educativo y del mundo laboral, porcentaje de niños en el nivel de pobreza o mortalidad infantil de 1 a 14 años, entre otros, que muestran grandes desigualdades en la distribución de la pobreza infantil.

6. Se empieza a entender el espacio- tiempo como el receptáculo de todas las cosas, ya no como dos realidades independientes, sino inextricablemente entrelazados, como dimensiones de un mismo continuo, por lo que los indicadores de uso temporal se hacen imprescindibles para captar las características socioespaciales. Surgen estudios que intentan medir estacionalidad, ritmos y frecuencias en el uso de los espacios. Encuestas de algunas administraciones ya incluyen este 
DISCAPACITADOS POR 100.000 HAB. DE CADA GRUPO DE EDAD. ESPAÑA, 1987

\begin{tabular}{|c|c|c|}
\hline & Media nacional & 14.979 \\
\hline $25-34 \quad$. & ....... & 4.644 \\
\hline $35-44 \quad .$. & ........................ & 6.368 \\
\hline $45-54 \quad .$. & ........................ & 13.969 \\
\hline $55-64 \quad$. & $\ldots \ldots \ldots \ldots \ldots$ & 27.814 \\
\hline $65-69 \quad$. & $\ldots \ldots \ldots \ldots \ldots \ldots \ldots \ldots \ldots$ & 40.164 \\
\hline $70-74 \ldots$ & (n. & 53.450 \\
\hline $75-79 \quad$. & 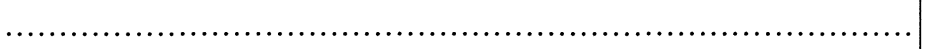 & 63.455 \\
\hline $80-84 \ldots$ & 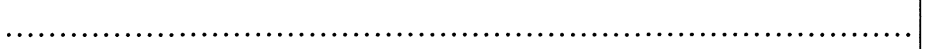 & 75.522 \\
\hline $85 y+$. & & 83.497 \\
\hline
\end{tabular}

I.N.E.: Encuesta sobre discapacidades, deficiencias y minusvalías. 1987.

tipo de datos. De esta nueva preocupación científica y política nace la necesidad de establecer indicadores que permitan medir las diferencias en el uso espacio-temporal según sexo, edad o zona de residencia (rural/urbano/metropolitano).

7. El nivel «micro» se presenta cada vez más necesario, sin olvidar la posición de los espacios en una escala macro o global, como ha sucedido en la casi totalidad de las ciencias, que buscan indistintamente en ambos extremos, en lo microscópico y en lo cósmico. Por eso se analiza cada vez más microespacios, lo «neo-local» y los microdatos (por ejemplo microeconómicos); se utilizan técnicas de microsimulación (para ensayar efectos de cambios en gasto social, por ejemplo); a la vez son más concretos los subgrupos de población analizados (gitanos, minusválidos, negros caribeños, etc.) ${ }^{5}$.

Por otra parte, se empiezan a obtener datos y a realizar análisis de muestras con individuos anónimos a nivel micro (Openshaw y Turton, 1996). También en la escala temporal se desciende a los niveles mínimos de análisis (por ejemplo, índices epidémicos de enfermeda-

5 Por ejemplo, es necesario descender a cada grupo étnico y sus composiciones sociales a nivel de barrio, para poder entender la segregación espacial londinense y la interacción entre las variables etnias y grupo social (Petsimeris, 1995). 
des de declaración obligatoria por semanas y días). Parece ser la era de los micro-datos, micro-análisis, micro-espacios, micro-censos, micro-simulación, y precisamente por ello surge la necesidad de datos e indicadores apropiados a dicha escala (Fotheringham, 1996).

8. Otra importante novedad se refiere a la forma de tratamiento $y$ de acceso a los datos. La informatización de la información ha hecho factible el análisis conjunto de gran cantidad de variables referidas a multitud de espacios, y ha facilitado el paso de indicadores simples a otros más complejos. La difusión de los datos, que normalmente se obtenían en soporte papel, ahora puede alcanzarse muchas veces en soporte magnético. Se distribuyen así múltiples bases de datos estadísticos, como por ejemplo WISTAT (Women's Indicators and Statistics, de Naciones Unidas), STARS (World Bank's Socioeconomic Time-Series Access and Retrieval System), las de la O.C.D.E. y muchas otras referidas a características nacionales y regionales.

\section{Nuevos indicadores sintéticos a nivel mundial}

Desde hace unos años se han venido difundiendo nuevos indicadores sintéticos. Presentan la ventaja de favorecer las comparaciones en contextos amplios.

Índice de desarrollo humano (IDH).-El Programa de las Naciones Unidas para el Desarrollo (PNUD) elabora desde 1990 un informe anual sobre desarrollo humano, aportando este nuevo índice.

El IDH se basa en tres indicadores: longevidad (esperanza de vida al nacer), nivel educativo (medida ponderada de alfabetización y tasas de escolarización primaria, secundaria y terciaria), y nivel de vida (P.I.B. per cápita).

Las clasificaciones del índice de desarrollo humano no siempre se emparejan con las clasificaciones de ingreso. Treinta y siete países tenían en 1993 una ubicación en el IDH que era 20 puntos superior o inferior a su clasificación por el ingreso per cápita, lo que indicaba que la correlación entre ingreso y desarrollo humano distaba mucho de ser perfecta en muchos países ${ }^{6}$. Los mayores valores de IDH co-

\footnotetext{
${ }_{6}$ Este indicador también se ha utilizado para medir diferencias entre grupos de población dentro de un país, por ejemplo en Méjico el IDH de la población indígena es sólo 0.700, mientras que para el resto de la población es 0.890 (PNUD, 1996).
} 
rrespondían en 1993 a Canadá, Estados Unidos, Japón y Países Bajos. España ocupaba la décima posición.

Índice de sufrimiento humano (ISH).-Population Action International en 1992 valoraba estadísticamente las condiciones de vida en 141 países, elaborando un indicador sintético que compila 10 medidas de bienestar humano muy diversas: esperanza de vida, suministro calórico diario, derechos civiles, acceso a agua pura, inmunización infantil, PNB per cápita, tasa de inflacción, tasa de teléfonos por mil habitantes y grado de libertad política.

Según este indicador había 24 países industrializados, que encerraban el $15 \%$ de la población mundial (797 millones), con sufrimiento humano mínimo, mientras que 3.500 millones de habitantes padecían sufrimiento humano alto y 433 millones, correspondientes a 27 países, tenían sufrimiento extremo. España se encontraba en el privilegiado grupo de sufrimiento humano mínimo, con el decimoctavo lugar. Según este indicador la mejor situación correspondía a Dinamarca y Países Bajos y las peores posiciones eran ocupadas por Mozambique, Somalia y Afganistán (Cuadro IV).

\section{Cuadro IV}

DISTRIBUCIÓN DE LA POBLACIÓN MUNDIAL SEGÚN ÍNDICE DE SUFRIMIENTO HUMANO, 1992

\begin{tabular}{l|c|c}
\hline \multicolumn{1}{c|}{ Valor del indice } & N.o de países & \% Población mundial \\
\hline$<$ 25. Mínimo & 24 & 15 \\
25-49. Moderado & 34 & 12 \\
50-74. Alto & 56 & 65 \\
75 y +. Extremo & 27 & 8 \\
\hline
\end{tabular}

Fuente: Population Action International. 1992.

Índice de pobreza de capacidad (IPC).-Es un complemento del índice de pobreza de ingreso (PIB per cápita). Como ha señalado el Banco Mundial, los indicadores económicos basados tanto en PIB como en PNB no pueden lograr una estricta comparabilidad internacional, ni miden el grado de bienestar ni el éxito en el desarrollo. Naciones 
Unidas mide el indice de pobreza de incapacidad por primera vez en el Informe de Desarrollo Humano de 1996, como una nueva medición de la privación humana. En el IPC se tiene en cuenta la falta de tres capacidades básicas: a) estar bien alimentado y sano, representada por la proporción de niños menores de cinco años que tienen peso insuficiente, b) capacidad de procreación en condiciones saludables, indicada por la proporción de nacimientos sin asistencia de personal sanitario, c) capacidad de obtener educación y conocimientos, expresada mediante el nivel de la alfabetización femenina (Cuadro V).

Cuadro V

POBLACIÓN CON POBREZA DE CAPACIDAD Y POBREZA DE INGRESO, 1993. \%

\begin{tabular}{|c|c|c|}
\hline País & $\begin{array}{c}\text { \% Pobreza } \\
\text { de capacidad }\end{array}$ & $\begin{array}{l}\text { \% Pobreza } \\
\text { de ingreso }\end{array}$ \\
\hline Bangladesh ..... & 79,9 & 47,5 \\
\hline India............ & 61,5 & 25,4 \\
\hline Pakistán ....... & 60,8 & 34,0 \\
\hline Guinea-Bissau $\ldots . . \ldots \ldots \ldots \ldots$ & 56,6 & 49,0 \\
\hline Marruecos ...................... & 49,7 & 13,1 \\
\hline Uganda ........................ & 45,9 & 55,0 \\
\hline Indonesia $\ldots . . . . . . . . . . . . . . . .$. & 42,3 & 16,7 \\
\hline Ghana ....................... & 39,3 & 35,9 \\
\hline Kenya ............................ & 33,8 & 37,0 \\
\hline Túnez ........................... & 29,9 & 14,1 \\
\hline Perú ............................. & 25,7 & 32,0 \\
\hline Zimbabwe ...................... & 22,3 & 25,5 \\
\hline Tailandia ...................... & 21,1 & 21,8 \\
\hline Sri Lanka ........................ & 19,3 & 22,4 \\
\hline China $\ldots \ldots \ldots \ldots \ldots \ldots \ldots \ldots \ldots$ & 17,5 & 10,9 \\
\hline
\end{tabular}

Fuente: PNUD. Informe sobre desarrollo humano. 1996.

Índice de riesgo reproductivo.-Fue calculado por Population Action International, en 1995, para 118 países que representaban el $94 \%$ de la población mundial. No es sólo un indicador demográfico y sanitario, sino que tiene interés como indicador de género. Está formado

$$
-703-
$$


por la combinación de 10 indicadores de salud maternal y sexual de las mujeres : procreación temprana (número anual de nacimientos por cada 100 mujeres de 15 a 19 años de edad), tasa de prevalencia anticonceptiva, políticas oficiales de aborto, prevalencia de anemia en embarazadas, acceso a la atención de salud durante el embarazo y parto, prevalencia de infecundidad primaria, prevalencia de infección por VIH en mujeres de bajo riesgo, riesgo de muerte en la maternidad. En esta misma línea existen cálculos de tasas de mutilación genital femenina, referidos a varios países africanos.

Índice de educación femenina.-Realizado por Population Action International, sobre 112 países con una población conjunta de 5.200 millones de habitantes. Incluye grado de instrucción de la mujer, relación entre la matrícula primaria y secundaria femenina y masculina, la tasa bruta de matriculación primaria y secundaria femenina. Es una medida que expresa el estado de la nivelación de géneros.

Índice de desarrollo relativo al género (IDG).-También denominado Indice de desarrollo relacionado con la mujer, fue introducido por Naciones Unidas en el Informe sobre Desarrollo Humano de 1995, combina las mismas variables que el IDH, pero se ajusta para incluir la desigualdad de género (Cuadro VI).

Cuadro VI

DISPARIDAD DE GÉNERO

\begin{tabular}{|c|c|c|c|}
\hline & $\begin{array}{l}\text { Categoría } \\
\text { IDG }\end{array}$ & $\begin{array}{l}\text { Categoría } \\
\text { IDH }\end{array}$ & $\begin{array}{c}\text { Categoría IDH menos } \\
\text { categoría IDG * }\end{array}$ \\
\hline Suecia $\ldots \ldots \ldots \ldots \ldots \ldots \ldots \ldots$ & 1 & 9 & 8 \\
\hline Francia ......................... & 8 & 7 & -1 \\
\hline España $\ldots \ldots \ldots \ldots \ldots \ldots \ldots \ldots$ & 20 & 10 & -10 \\
\hline Chile ........................... & 44 & 29 & -15 \\
\hline 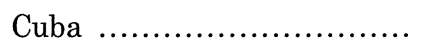 & 59 & 64 & 5 \\
\hline Arabia Saudí ................. & 85 & 55 & -30 \\
\hline Zaire ......................... & 109 & 110 & 1 \\
\hline Etiopía ..................... & 132 & 132 & 0 \\
\hline
\end{tabular}

* Categoría ordena los valores resultantes de mejor a peor. Una diferencia positiva en la resta de categorías IDH-IDG indica que en ese país se obtienen resultados mejores respecto de la igualdad de género que respecto de los adelantos en general. 
Índice de potenciación de género (IPG).-También se introdujo en el Informe sobre Desarrollo Humano de 1995 de Naciones Unidas, e incluye variables para la medición de la potenciación relativa. Se centra en el grado de participación y mide la desigualdad de género en esferas clave económicas y políticas. Se forma con los siguientes indicadores: porcentaje de mujeres en escaños parlamentarios, en puestos ejecutivos y de gerencia, en puestos profesionales y técnicos, y participación en el ingreso proviniente del trabajo.

\section{El valor de los indicadores}

Mucho ha progresado la Geografía en la elaboración de cartografía, en determinadas técnicas, o en algunos aspectos específicos, pero poco se ha logrado en el tema de los sensores socioespaciales fiables y sensibles, existiendo aún una gran pobreza de conceptos, terminología y cálculo de indicadores.

Hérin (1984) dividía el campo de la Geografía Social en 4 dominios principales: indicadores sociales, las cuestiones sociales, los grupos sociales y las combinaciones socio-espaciales. Algo se ha avanzado en los tres últimos dominios, pero se ha olvidado un poco el primero, cuando no sólo es un ámbito de investigación interesante en sí mismo, sino que es el que procura los instrumentos que pueden favorecer el conocimiento detallado y profundo de los restantes dominios y ayuda a comprender el objeto complejo que son «les réalités socio-spatiales», las formaciones socio-espaciales (Chevalier y $\mathrm{Bu}$ leon, 1986) y el papel de cada espacio y sus hombres en el gran puzzle de la globalización.

Es preciso traer del destierro a los indicadores. Son retos inminentes, que debe asumir la Geografía Social, el conocer todos los indicadores existentes (tanto sociales como microeconómicos o sanitarios), el recurrir a los indicadores de sustitución cuando los directos no fueran posibles, realizar su análisis crítico, perfeccionar algunos de ellos si fuera factible, crear nuevos indicadores sintéticos socioespaciales y no limitarse a utilizar los propuestos por economistas y sociólogos. Por ejemplo, los geógrafos deberían intentar aportar algo a la demanda de indicadores en relación con las Agendas Locales XXI que tienen como objetivo lograr ciudades sostenibles y saludables.

$$
-705-
$$


Por otra parte, también es necesario continuar presionando y solicitando a las instituciones productoras de estadísticas datos con las características, ritmo temporal y desagregación espacial apropiados, ya que los organismos generadores de estadísticas simples e indicadores sintéticos sólo permiten trabajar con divisiones espaciales administrativas o delimitadas por el poder político o económico, y condicionan las escalas temporales y espaciales de las investigaciones, limitando sus objetivos y resultados. No hay que olvidar que la selección de escala puede ser en sí misma la elección de un enfoque.

Utilizar indicadores e intentar crearlos no significa luchar por «new markets in Knowlegde», ni por mercantilizar más fácilmente los resultados de la investigación, sino simplemente intentar analizar la situación de justicia socio-espacial con estas herramientas y todas las otras que estén disponibles, como documentación derivada de grupos de discusión (focus group), historias de vida, observación, etc. Se pueden usar las mismas armas con diferentes fines y hacia diferentes frentes.

No es conveniente que la creación y selección de indicadores surja solamente del cerrado mundo académico o del poder político, sino que se debería contar con la participación ciudadana, como ya ha sucedido en el caso de los indicadores de sostenibilidad de Seattle (EE.UU.) ${ }^{7}$. Por otra parte, los propios indicadores pueden ser un factor de concienciación ciudadana, rompiendo a veces la pasividad con la fuerza de los datos contundentes que promueven la toma de conciencia colectiva de un problema.

Profundizar en microvariables, microparámetros y microespacios procurará un minucioso análisis, pero la agregación de los resultados puede no ser siempre viable. Lo importante es avanzar en la comprensión de las interacciones socioespaciales, bien llegando a generalizaciones o aportando análisis cuantitativos y cualitativos de lo que le sucede «a esta pequeña muestra, aquí y ahora» sin la menor intención de generalización, como proponen muchos encuadres postmodernos.

Los indicadores no sólo permiten conocer el estado de unas variables en un espacio dado, sus diferencias por grupos poblacionales

7 Allende, J. (1995), «Desarrollo sostenible. De lo global a lo local». Ciudad y Territorio, III, 104, 267-281. 
o sociales, sino también posibilitan la comparación de sus valores en contextos más amplios espaciales, evolutivos y rítmicos. Pueden ayudar a entender permanencias y mutaciones, duración, continuidad y descontinuidad, la intensidad de los fenómenos y de los cambios y su velocidad, la posible incidencia de factores endógenos y exógenos.

Por otra parte, la influencia innegable que ejercen sobre lo local hechos lejanos o globales (que no podemos medir ni observar directamente, ni extraer del trabajo de campo tradicional), nos obliga a recurrir cada vez más a indicadores internacionales, por ejemplo, los que aportan información sobre la nueva división internacional del trabajo.

Los indicadores cuantitativos y cualitativos facilitan la inducción y la búsqueda de correlaciones, permiten observar tendencias, evaluar secuencias, detectar diferencias espaciales y algunas interacciones socioespaciales, realizar análisis prospectivos, denunciar procesos indeseables en fase incipiente, marcar los puntos negros prioritarios para la toma de decisiones políticas y de planificación, revisar el efecto o impacto de la aplicación de algunas medidas o programas (monitorización de efecto de políticas fiscales, de protección social, de ayuda humanitaria, etc.), realizar análisis retrospectivos, e incluso promover la participación ciudadana y facilitar datos básicos a los movimientos contra la segregación o discriminación. De ahí el interés de su discusión.

\section{BIBLIOGRAFÍA}

Abellan, A. (1994): «La Conferencia Internacional sobre Población y Desarrollo. El Cairo, 1994», Estudios Geográficos, 217, 743-753.

Abellán, A. (1995): «La Cumbre Mundial sobre desarrollo social. Copenhague. 1995». Estudios Geográficos, 218, 175-178.

Abellan, A. (Coord.) (1996): Envejecer en España. Manual estadistico sobre el envejecimiento de la población. Madrid. Fundación Caja de Madrid, 74 pp.

Álvarez URí, F. et al. (1995): Desigualdad y pobreza hoy. Madrid. Talasa, 203 pp.

Banco Mundial (1990): Informe sobre desarrollo mundial 1990. La pobreza. Washington.

BANCo Mundial (1993): Informe sobre el desarrollo mundial 1993. Invertir en salud. Washington.

Banco Mundial (1995): El mundo del trabajo en una economia integrada. Informe sobre el desarrollo mundial 1995. Washington.

Banco Mundial (1995): Social Indicators of Development. 1995. Baltimore and London. The Johns Hopkins University Press. 
CiREs (1994): La realidad social en España 1993-94. Bilbao Bizkaia, Fundación BBV, Caja de Madrid, 1042 pp. y 4 diskettes.

Chevalier, J.; Buleon, P. (1986): «Les concepts de la Géographie Sociale. Débat autour du concept de formation-spatiale». Géographie Sociale, 3, 7-18.

Eunostat (1994): Poverty statistics in the late 1980s: Research based on micro data. Luxembourg.

Eurostat (1994): Demographic statistics.

FOESSA (1994): V Informe sociológico sobre la situación social en España. Tomos I y II.

Fotheringham, A. S.; Pellegrini, P. A. (1996): «Microdata for migration analysis: A comparison of sources in the US, Britain and Canada», Area, 28, 3, 347-357.

GaRCía RAMón, M. D. (1989): "Para no excluir del estudio a la mitad del género humano: un desafío pendiente en Geografía Humana». Bol. Asoc. Geógrafos Españoles, n. ${ }^{\circ} 9$, $27-48$.

Geddes, J.; Muir, K. (1987): Aspects of Social Geography: change and development. London. Edward Arnold. 1987.

GREGORY, D.; URRY, J. (1985): Social Relations and spatial structures. London. Macmillan.

Hansluwka, H. E. (1985): «Measuring the health of populations, indicators and interpretations». Soc. Sci. Med, vol. 20, n. ${ }^{\circ} 12,1207-1224$.

Herin, R. (1984): Références, champ et méthodes de la Géographie Sociale. En: FREMonT, A.: Géographie Sociale. Paris, Masson. 121-160.

Horn, R. V. (1993): Statistical Indicators for Economic and Social Sciences. Cambridge University Press.

I.N.E. (1991): Indicadores sociales. Madrid. 365 pp.

I.N.E. (1993): Encuesta de presupuestos familiares 1991

I.N.E. (1993): Encuesta sociodemográfica. 1991.

I.N.E. (1994): Panorámica social de España. Madrid. 841 pp.

I.N.E. (1995): Defunciones según causa de muerte 1991. Tomos I y II.

I.N.E. (1995): Encuesta de morbilidad hospitalaria 1992. Madrid. 286 pp.

I.N.E. (1996): Anuario Estadistico. Madrid. 890 pp.

I.N.E. (1997): Indicadores sociales de España. Disparidades provinciales. Madrid. 641 pp. y 1 diskette.

MCDowell, L. (1991): «Life without Father and Ford: the new gender order of post-Fordism». Transactions, Institute of British Geographers, 16, 400-419.

McDowell, L. (1993): «Space, place and gender relations: Part I. Feminist empiricism and the Geography of social relations». Progress in Human Geography, 17,2, 157-179.

M.S.C. (1993): Indicadores de salud. Madrid. 305 pp. y 1 diskette.

M.S.C. (1994): Sistema Nacional de Salud. Servicios de Salud. Indicadores. 1993. 263 pp.

M.S.C. (1995): Encuesta Nacional de Salud de España. 1993. Madrid. 84 pp. y 1 diskette.

Mootz, M. (1986): «Health indicators». Soc. Sci. Med., vol. 22, n. ${ }^{\circ}$ 2, 255-263.

NACIONEs Unidas (1989): 1989 World Survey on the role of women in Development. Nueva York.

NaCiones Unidas (1989): Manual de indicadores sociales. Nueva York.

NACIONES UNIDAS (1995): Situación de la mujer en el mundo. 1995. Tendencias y estadísticas. Nueva York.

Nossikov, A. et al. (1990): «Using regional indicators: the experience of Europe». Wld. hlth. statist. quart. 43, 25-30.

O.C.D.E. (1995): Health Data 1995. Paris.

Olivera, A.; Abellán, A. (1994): «Les obstacles physiques de la cité: la brutalité de l'espace construit». Gérontologie et société., n. ${ }^{\circ}$ 69, 82-91.

Olivera, A. (1993): Geografía de la salud. Madrid. Síntesis.

Olivera, A. (1996): «Población, salud y bienestar». Treballs de la Societat Catalana de Geografia, n. ${ }^{\circ} 41$, vol. XI, 135-171. 
Olivera, A. (1996): La Cuarta Conferencia Mundial sobre la Mujer. Estudios Geográficos, n. ${ }^{\circ} 222,151-155$.

Openshaw, S.; TuRTON, I. (1996): «New opportunities for geographical census analysis using individual level data». Area, 28, 2, 167-176.

O.M.S. (1995): World Health Statistics. Annual. Ginebra.

Petsimeris, P. (1995): «Une méthode pour l'analyse de la division ethnique et sociale de l'espace intra-métropolitain du Grand Londres». L'Espace Géographique, 2, 139-153.

Picheral, H. (1976): Espace et santé. Géographie médicale du Midi de la France. Montpellier. Paysan du Midi.

PNUD (1996): Informe sobre desarrollo humano 1996. Madrid. Naciones Unidas. Mundi Prensa.

Puyol, R. (Ed.) (1997): Dinámica de la población en España. Madrid. Ed. Síntesis, 399 pp.

Sмiтн, D. M. (1980): Geografía Humana. Barcelona. Oikos-Tau, 586 pp.

Sмiтн, S. J. (1990): «Social Geography: patriarchy, racism, nationalism». Progress in Human Geography, vol. 14, n. ${ }^{\circ}$ 2, 261-271.

WatTs, M. (1989): «La Geografía y la lucha en defensa del medio ambiente: la integración de la ecología humana y la economía política, o ¿Marx era rojo y verde?». Bol. Asoc. Geógrafos Españoles, n. ${ }^{\circ}$ 9, 109-126.

RESUmen: Indicadores en Geografía Social. Reflexión y propueta de debate sobre el valor y la necesidad de los indicadores en Geografía Social. Se presenta una tipología, los factores recientes que inducen a la creación de indicadores y nuevos indicadores sintéticos de nivel mundial.

Palabras Clave: Geografía Social. Indicadores sociales. Bienestar. Desarrollo. Población.

AвSTRACT: Indicators in Social Geography. This paper examines the more general background to social indicators. It discusses possible purposes for wich social indicators should be developed. Social indicators have to be seen as a tool to be used in planning and monitoring strategies and to illustrate the differences in social situations within countries or regions. A typology and new complex indicators are presented.

KEY words: Social Geography. Social indicators. Welfare. Development. Population.

Résumé: Indicateurs en Gégraphie Sociale. La réflexion qui suit s'inscrit dans le nécessaire débat sur les indicateurs en Géographie Sociale et son utilité. On y pesente une typologie, les récents intérêts thématiques et les nouveaux indicateurs synthétiques de niveau mondial. lation.

Mots CLÉ: Géographie Sociale. Indicaterus sociaux. Développement. Bien-être. Popu- 